\title{
Queuing Analysis in Multichannel Cognitive Spectrum Access: A Large Deviation Approach
}

\author{
Amine Laourine, Shiyao Chen, and Lang Tong \\ School of Electrical and Computer Engineering \\ Cornell University, Ithaca, NY 14853 \\ Email: $\{$ al496,sc933,1t35\}@ cornell.edu
}

\begin{abstract}
The queueing performance of a (secondary) cognitive user is investigated for a hierarchical network where there are $N$ independent and identical primary users. Each primary user employs a slotted transmission protocol, and its channel usage forms a two-state (busy,idle) discrete-time Markov chain. The cognitive user employs the optimal policy to select which channel to sense (and use if found idle) at each slot. In the framework of effective bandwidths, the stationary queue tail distribution of the cognitive user is estimated using a large deviation approach for which closed-form expressions are obtained when $N=2$. Upper and lower bounds are obtained for the general $N$ primary user network. For positively correlated primary transmissions, the bounds are shown to be asymptotically tight. Monte Carlo simulations using importance sampling techniques are used to validate the obtained large deviation estimates.
\end{abstract}

Index terms-Effective bandwidth, Queueing analysis, Cognitive radio and Dynamic spectrum access.

\section{INTRODUCTION}

Cognitive radio is an emerging technology aimed at improving spectrum efficiency by allowing cognitive users to discover and capture transmission opportunities. For a hierarchical network where a cognitive user is secondary to spectrum incumbents (the primary users), the cognitive user may at times avoid transmission to limit its interference to the primary users. The achievable Quality of Service (QoS) of cognitive communications is therefore dictated by the traffic of the primary users as well as the access policy used by the cognitive user to locate spectrum vacancies.

While the potential of cognitive communications in maximizing spectrum utilization is widely recognized, it is not well understood what types of applications are suitable for cognitive communications. Can an application with a certain required QoS be supported by the cognitive user? Can the cognitive user provide enough "bandwidth" for video streaming, voice over IP, or online gaming? These are the motivating questions behind the problems considered in this paper.

We consider a hierarchical cognitive network where a cognitive (secondary) user exploits transmission opportunities in a multichannel communication setting [1], [2]. In particular, we assume the presence of $N$ parallel channels, each assigned to a primary user. The transmissions of primary users are bursty and statistically independent, and they are modeled

This work is supported in part by the National Science Foundation under Contract CCF-0635070 and the Army Research Office MURI Program under award W911NF-08-1-0238. as Markovian on-off processes, with the "off" periods representing potential opportunities for the cognitive user. The cognitive user identifies transmission opportunities through channel sensing. We assume that the cognitive user can only sense one channel in each time slot. If the sensed channel is idle (i.e. the primary user is "off"), the cognitive user can transmit some of the data accumulated in its queue. If the sensed channel is busy, it has to wait until the next slot and sense again; opportunities in channels that are not sensed are lost.

Because of the lack of access priority, cognitive transmissions are intermittent. Thus queueing for the cognitive user plays a crucial role. If the opportunities are scarce, a source with a high traffic rate will result in excessive packet drops or queue instability. To determine whether an application is admissible by the cognitive user, it is necessary to characterize the relationship between the traffic arrival rate and the queue distribution. In particular, it is of practical significance to determine the maximum traffic arrival rate that can be supported by a cognitive user provided that the probability of its queue length exceeding a certain level is bounded above by some prescribed $\epsilon$. In this context, the queue distribution determines the QoS characteristics, and the associated maximum arrival rate can be interpreted as the effective bandwidth available for cognitive communications.

\section{A. Summary of Results and Contributions}

The objective of this paper is to obtain an analytical characterization for the relationship between the arrival rate of the cognitive traffic and the queue distribution of the cognitive user. To this end, we adopt the large deviation approach in the framework of effective bandwidth analysis. For a review, see [3] and references therein. In this analysis, we are interested in obtaining the large deviation approximation of the queue length distribution, which is a function of the primary users' traffic and also a function of the cognitive access protocol. In this paper, we analyze the myopic access protocol of Zhao, Krishnamachari, and Liu (ZKL) [4]. The main reason to study $\mathrm{ZKL}$ is that, for independent and identically distributed primary users, ZKL is throughput optimal under the assumption of positively correlated Markovian primary traffic [4], [5]. Also from a practical stand point, ZKL has a simple structure that is easy to implement, analyze, and insensitive to traffic parameters. 
The key step in our large deviation analysis is obtaining a certain Gärtner-Ellis limit, which is often not available in closed form. The specific structure of the ZKL protocol makes it possible to transform the problem to the large deviation analysis of a certain sequence from a countable state Markov chain. For the two channel case, this Gärtner-Ellis limit can be obtained in closed form. From there, we obtain the large deviation estimate of the stationary queue distribution $Q_{\infty}$. Specifically, given the arrival rate $a$ and the traffic parameters of the primary users, under stability conditions, the following identity holds

$$
\lim _{q \rightarrow \infty} \frac{1}{q} \log \operatorname{Pr}\left(Q_{\infty}>q\right)=-\theta^{*}(a)
$$

where $\theta^{*}(a)>0$ represents the decay rate of the probability of the buffer overflow if the queue size is large enough. In this paper, we obtain analytical characterizations of $\theta^{*}(a)$ for $N=2$ primary channels, see Corollary 1 , which provides the connection between the arrival rate $a$ and the queue length $Q_{\infty}$ in the large queue length regime. For $N>2$ primary channels, we do not have a similar closed-form expression. However, we obtain closed-form upper and lower bounds for the Gärtner-Ellis limit, which gives the corresponding bounds on $\theta^{*}(a)$. We show that, for positively correlated Markovian primary traffic, the bounds are exponentially tight as $N \rightarrow \infty$.

A relevant issue of the large deviation analysis is how "large" is large enough. We address this issue through simulation of rare events using the importance sampling technique. It is significant that our simulation shows that the large deviation approximation of the queue distribution is sufficiently accurate with a moderate queue size, see Section IV.

\section{B. Related Work}

The methodology for queueing analysis used in this paper comes from the effective bandwidth theory (see Glynn and Whitt [6], Veciana and Walrand [8], Elwalid and Mitra [9], and Kelly [3]). A substantial body of literature exists for the analysis of queue distributions of various multiplexed access schemes, some of the earlier work for wireline networks can be found in [9], [10], [11], [12]. The cognitive access scheme considered in this paper results in random departures from the queue of the cognitive user, a phenomenon similar to fading channels. There has been recent work considering such scenarios. See, e.g., [13], [14], [15], [16].

A great deal of effort has been devoted to obtaining cognitive access schemes that are throughput optimal (see [1] for a recent survey on access protocols). The work most relevant to this paper is [4] where the authors proposed the (ZKL) protocol and established the throughput optimality for the two channel case (see also [5]). Their approach points to the analysis of a key Markov chain that models the transmission periods. In this paper, it is the large deviation property of the same Markov chain that leads to the analytical characterization of the Gätner-Ellis limits.

Despite its importance, the analysis of delays and queuing properties for cognitive communications is scarce. In [17], the authors present queuing analysis of a multichannel hierarchical cognitive network with multiple secondary users. While the network setting in [17] is more general, the aim and technique used in [17] are different from ours. The results of [17] are about the moments of the queue lengths of secondary users, while we consider the large deviation approximation of the queue distribution. The authors in [18] have considered the decay rate of the queue of the cognitive user. However, in their problem, the cognitive user has access to only one channel, and the primary user transmission pattern (i.e. busyidle transitions) is assumed to be independent from slot to slot whereas, in our paper, the cognitive user has access to an arbitrary number of channels (which he visits according to the ZKL protocol) and the primary users activity is assumed to be Markovian. In [19], the decay rate of the queue of the CU is also considered, the cognitive user has access to multiple channels and the primary user has a Markovian activity. The major difference between [19] and our work is that in [19] the cognitive user knows perfectly the idle channels whereas in this paper this is not the case and the user discovers the idle channels through an access scheme. The same remark holds for [20] as they assume that a base station plays the role of a scheduler and assigns idle channels to secondary users. The analysis in [19] and [20] can only provide an optimistic upper bound on the queuing performance since they assume a perfect knowledge of the spectrum opportunities. In this paper we avoid this problem by examining the combined effect of the access scheme and the primary users traffic on the queueing performance of the CU.

\section{Problem Description}

We first present a description of our model and assumptions. The $N$ primary users each can only transmit in its own channel according to time slots. The transmission activities on the $N$ channels are modeled as $N$ independent and identically distributed two state (busy or idle) Markov chains, and the cognitive user can only transmit in idle slots. We assume that $p_{11}=\operatorname{Pr}[$ idle $\rightarrow$ idle $]$ and $p_{01}=\operatorname{Pr}$ [busy $\rightarrow$ idle]. When $p_{11} \geq p_{01}$ the channel is said to be positively correlated and when $p_{11}<p_{01}$ we speak of negative correlation.

In each time slot the cognitive user can only sense one of the $N$ channels. If the sensed channel is idle and the cognitive user has data in its queue, the cognitive user transmits and the transmission is assumed to be successful. If the channel is busy, the cognitive user does not transmit and the slot is lost. The method used to select which channel to sense in each slot is an important aspect of the problem and will be detailed later. The incoming traffic of the cognitive user is assumed to be a constant arrival process with an intensity of $a$ bits $^{1}$ per slot and the arrived bits are stored in a buffer of size $b \gg 1$.

For a fixed access policy $\pi$, let $Q_{n}^{\pi}$ denote the queue size at the end of the $n$th slot. Then $\left(Q_{n}^{\pi}\right)_{n \geq 0}$ is a Lindley process satisfying the following recursion

$$
Q_{n}^{\pi}=\left(Q_{n-1}^{\pi}+a-C_{n}^{\pi}\right)^{+}, \quad n \geq 1, \quad Q_{0}^{\pi}=0,
$$

\footnotetext{
${ }^{1}$ Note that the choice of bits as the data traffic units is arbitrary.
} 
where $(x)^{+}$is given its usual meaning $\max (x, 0)$, and $C_{n}^{\pi}$ represents the output process of the queue and is equal to zero if the channel sensed at the $n$th slot is busy and equal to $c$ bits if the channel is idle. Under stability conditions, $Q_{n}^{\pi}$ converges in distribution to $Q_{\infty}^{\pi}$. In this paper, we are interested in characterizing the tail distribution of $Q_{\infty}^{\pi}$. From now on, we shall omit the superscript $\pi$.

\section{A. Effective Bandwidth and Effective Capacity}

We state first a well known result in effective bandwidth analysis (see for example [6] and [11]). The following lemma characterizes the decay rate of the steady state tail distribution of the queue.

Lemma 1. Assume that the queue is stable and that $\left(C_{n}\right)_{n \geq 1}$ satisfies the Gärtner-Ellis limit, i.e., there exits a differentiable function $\Psi_{C}(\theta)$ such that

$$
\lim _{n \rightarrow \infty} \frac{\log \mathrm{E}\left[\exp \left(\theta \sum_{k=1}^{n} C_{k}\right)\right]}{n}=\Psi_{C}(\theta) .
$$

Assume also that there exists a unique solution ${ }^{2} \theta^{*}(a)>0$ of the equation

$$
a \theta+\Psi_{C}(-\theta)=0 .
$$

Then it follows that $Q_{n} \Rightarrow Q_{\infty}$ (the symbol $\Rightarrow$ refers to convergence in distribution) and

$$
\lim _{x \rightarrow \infty} \frac{\log \operatorname{Pr}\left(Q_{\infty}>x\right)}{x}=-\theta^{*}(a) .
$$

This lemma implies that for a buffer of size $b$ large enough, the buffer overflow probability $P_{O} \triangleq \operatorname{Pr}\left[Q_{\infty}>b\right]$ can be approximated by $P_{O} \approx \gamma^{*} \exp \left(-\theta^{*} b\right)$. The constant $\gamma^{*}$ is in general difficult to obtain, but it has been suggested [7] that $\gamma^{*} \approx 1$ is in general a good approximation.

In the context of multichannel cognitive spectrum access, the available effective bandwidth (or effective capacity ${ }^{3}$ ) can be defined as the maximum arrival rate $a$ that can be supported provided that the buffer overflow probability satisfies $P_{O} \leq \epsilon$ for some prescribed QoS parameter $\epsilon$. Specifically, for a large buffer of size $b \gg 1$, the maximum sustainable arrival rate (effective bandwidth) $a^{*}(\epsilon)$ can be defined as

$$
a^{*}(\epsilon) \triangleq \max \left\{a: \exp \left(-b \theta^{*}(a)\right) \leq \epsilon\right\} .
$$

The following Lemma which connects the effective bandwidth $a^{*}(\epsilon)$ with the Gärtner-Ellis limit $\Psi_{C}(\theta)$ immediately follows from Lemma 1.

Lemma 2. Under the assumptions of Lemma 1, given $\epsilon$ and a queue size $b \gg 1$, the effective bandwidth is given by

$$
a^{*}(\epsilon)=b \frac{\Psi_{C}\left(\frac{\log \epsilon}{b}\right)}{\log (\epsilon)}
$$

\footnotetext{
${ }^{2}$ The decay rate $\theta^{*}(a)$ depends also on $c$, so a more appropriate notation would be $\theta^{*}(a, c)$. However we decided to drop the $c$ argument in order to emphasize the dependence on the arrival intensity $a$ which is, contrary to the capacity $c$, selected by the user.

${ }^{3}$ This is also referred to as the effective capacity in [13], [14], [15], [16].
}

As expected, as the buffer size $b \rightarrow \infty$ or as $\epsilon \rightarrow 1$, we have $a^{*}(\epsilon) \rightarrow E\left(C_{n}\right)$.

Another QoS metric that is also sometimes used is the delay violation probability, i.e., $\operatorname{Pr}\left[D_{\infty}>d\right]$, where $D_{\infty}$ represents the stationary delay experienced and $d$ is a maximum tolerable delay. A user may for instance require that $\operatorname{Pr}\left[D_{\infty}>d\right] \leq \xi$. For a constant arrival process, which is the case in this paper, this constraint can be reformulated (see for instance [15], [22]) as a constraint on the steady-state queue length. Consequently there is no loss in generality in considering the buffer overflow probability as the QoS metric.

The strength of Lemma 1 is that it allows general dependencies in $C_{n}$. However, it masks the difficulty in calculating the Gärtner-Ellis limit $\Psi_{C}(\theta)$. The key step in this paper is evaluating $\Psi_{C}(\theta)$ from which we will be able to assess the available bandwidth for cognitive communication given the traffic parameters of the primary user and the access protocol of the cognitive user.

\section{B. The single primary channel example}

Before presenting the general result, we consider the special case when there is only one primary user. In this case, the calculation of the effective bandwidth does not depend on the access protocol. Classical results from the theory of effective bandwidths allow us to easily compute the Gärtner-Ellis limit $\Psi_{C}(\theta)$. Here $C_{n}=c X_{n}$ where $X_{n}$ is the state of the on-off channel, $C_{n}$ is hence a Markov chain on the state space $\{0, c\}$ (it is also a special case of a Markov modulated process with respect to $\left.\left(X_{n}\right)_{n \geq 1}\right)$. The Gärtner-Ellis limit $\Psi_{C}(\theta)$ in this case is given in the following lemma (see for instance [21, pp. 244-246]).

Lemma 3.

$$
\Psi_{C}(\theta)=\log \left(\frac{\phi_{1}(\theta)+\sqrt{\phi_{1}^{2}(\theta)-4 \phi_{2}(\theta)}}{2}\right)
$$

with $\phi_{1}(\theta)=p_{00}+p_{11} e^{c \theta}$ and $\phi_{2}(\theta)=\left(p_{00}+p_{11}-1\right) e^{c \theta}$.

With such a closed-form expression, we can examine some special cases. For example, for very wideband channels, $c \gg 1$ and the effective bandwidth is given by

$$
a^{*}(\epsilon) \approx b \frac{\log \left(1-p_{01}\right)}{\log (\epsilon)} .
$$

Here we note that the effective bandwidth is proportional to the buffer size in the wideband regime. It is interesting that, in this regime, $a^{*}(\epsilon)$ depends mostly on the probability that the channel changes from busy to idle. This is because when $c \gg 1$, the queue is drained quickly when the channel is idle. The behavior of $a^{*}(\epsilon)$ can be easily interpreted for cases when $p_{01}$ is close to one or close to zero.

This digression gives a glimpse of the type of results that we are aiming for. In the next section we will treat this general case by providing a closed form expression for $\Psi_{C}(\theta)$ when $N=2$ and providing upper and lower bounds in the case $N>2$. 


\section{COMPUtATion OF THE GärTNER-Ellis Limit $\Psi_{C}(\theta)$}

We now focus on the evaluation of the Gärtner-Ellis limit that determines the effective bandwidth. This limit is a function of the primary users activity pattern (i.e., $p_{11}$ and $p_{01}$ ) as well as the cognitive access policy used to discover the spectrum opportunities. We consider here the ZKL policy [4], which is throughput optimal for positively correlated Markov primary traffic [5]. It is the special structure of ZKL that makes the computation tractable.

\section{A. The ZKL access policy}

The ZKL policy has a simple round-robin structure. For brevity, we describe here only the positively correlated case. See [4] for complete details. Prior to access, channels are ranked according to their prior probabilities of being idle; the highest ranked channel is the one most likely to be idle, the lowest ranked one the least. If such prior probabilities are not available, the channels are ranked arbitrarily. Starting at the highest ranked channel in the priority list, sense the channel and transmit for as many idle slots as possible. The first time the channel becomes busy, switch to the next highest ranked channel in the list and stay in this channel as long as it is idle. The first time the channel becomes busy, move down in the priority list until reaching the lowest ranked channel. After the lowest ranked channel becomes busy, the policy goes back to the highest ranked channel again. A sample path of access is illustrated in Fig. 1.

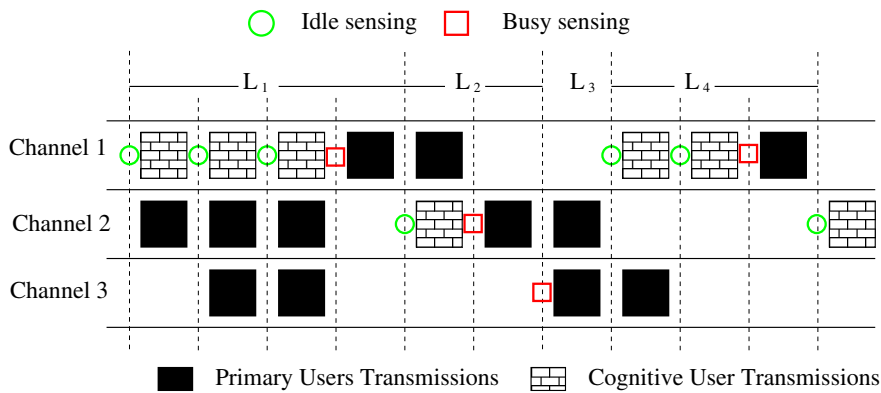

Fig. 1. Illustration of a sample path of the access policy.

Let $L_{k} \geq 1$ be the number of slots that the cognitive user stays in a channel during the $k$ th transmission period induced by ZKL (see Fig. 1). It is shown in [4] that the sequence $\left(L_{k}\right)_{k>1}$ is an $(N-1)$-th order Markov chain. Define $\vec{L}(n) \triangleq$ $\left(L_{1}, \ldots, L_{n}\right)$ and let $\vec{l}(n)$ be a realization of $\vec{L}(n)$.

For the positively correlated case $\left(p_{11} \geq p_{01}\right)$, let $\alpha \triangleq$ $p_{11}-p_{01}$ and $\beta \triangleq \frac{p_{01}}{p_{01}+p_{10}}$. We then have

$P\left[L_{n}=l_{n} \mid \vec{L}(n-1)=\vec{l}(n-1)\right]= \begin{cases}p_{00}^{\left(J_{n}\right)} & \text { if } l_{n}=1 \\ p_{01}^{\left(J_{n}\right)} p_{11}^{l_{n}-2} p_{10} & \text { if } l_{n} \geq 2\end{cases}$

where $J_{n}=\sum_{i=1}^{N-1} l_{n-i}+1, p_{01}^{\left(J_{n}\right)}=\beta\left(1-\alpha^{J_{n}}\right)$ and $p_{00}^{\left(J_{n}\right)}=$ $1-p_{01}^{\left(J_{n}\right)}$.
For the negatively correlated case $\left(p_{11}<p_{01}\right)$, the transition probability becomes

$P\left[L_{n}=l_{n} \mid \vec{L}(n-1)=\vec{l}(n-1)\right]=\left\{\begin{array}{ll}p_{11}^{\left(T_{n}\right)} & \text { if } l_{n}=1 \\ p_{10}^{\left(T_{n}\right)} p_{00}^{l_{n}-2} p_{01} & \text { if } l_{n} \geq 2\end{array}\right.$,

where $T_{n}$ is a certain function ${ }^{4}$ of $\vec{l}(n-1), p_{11}^{\left(T_{n}\right)}=\beta(1+$ $\left.\frac{p_{10}}{p_{01}} \alpha^{T_{n}}\right)$, and $p_{10}^{\left(T_{n}\right)}=1-p_{11}^{\left(T_{n}\right)}$.

A key quantity in our calculation is the Gärtner-Ellis limit of $L_{n}$ defined by

$$
\Psi_{L}(\theta) \triangleq \lim _{n \rightarrow \infty} \frac{\log \mathrm{E}\left[\exp \left(\theta \sum_{k=1}^{n} L_{k}\right)\right]}{n},
$$

which is simpler to evaluate than $\Psi_{C}(\theta){ }^{5}$

\section{B. Computing $\Psi_{C}(\theta)$ via $\Psi_{L}(\theta)$}

We will first establish an explicit relation between $\Psi_{L}(\cdot)$ and $\Psi_{C}(\cdot)$.

Theorem 1. The Gärtner-Ellis limit $\Psi_{C}(\cdot)$ is given by

$$
\Psi_{C}(\theta)=\left\{\begin{array}{ll}
c \theta-\Psi_{L}^{-1}(c \theta) & \text { if } p_{11} \geq p_{01}, \\
-\Psi_{L}^{-1}(-c \theta) & \text { o.w. }
\end{array} .\right.
$$

where $\Psi_{L}^{-1}(\cdot)$ denotes the inverse function of $\Psi_{L}(\cdot)$.

Proof: Let $S_{n}=\sum_{k=1}^{n} L_{k}, n \geq 1$ with $S_{0}=0$, also let $\{N(t): t \geq 0\}$ be the counting process associated to the sequence $\left(S_{k}\right)_{k \geq 1}$, i.e.,

$$
N(t)=\max \left\{k \geq 0: S_{k} \leq t\right\}, \quad t \geq 0 .
$$

We establish first a relation between $\Psi_{C}(\theta)$ and $\Psi_{N}(\theta)=$ $\lim _{t \rightarrow \infty} \frac{\log \mathrm{E}[\exp (\theta N(t))]}{t}$. Note that

$$
\mathrm{E}\left[e^{\theta \sum_{k=1}^{n} C_{k}}\right]=\sum_{l=0}^{\infty} \operatorname{Pr}[N(n)=l] \mathrm{E}\left[e^{\theta \sum_{k=1}^{n} C_{k}} \mid N(n)=l\right] .
$$

Also $\{N(n)=l\} \Leftrightarrow\left\{S_{l} \leq n<S_{l+1}\right\}$, consequently on the event $\{N(n)=l\}$ we have that $\sum_{k=1}^{n} C_{k}=c(n-l)$ when $p_{11} \geq p_{01}$, whereas if $p_{11}<p_{01}$ we have $\sum_{k=1}^{n} C_{k}=c l$. This is because for the positively correlated case, a channel switch corresponds to a slot without transmission and in the negatively correlated case the opposite is true.

Consequently, if $p_{11} \geq p_{01}$, we deduce

$$
\mathrm{E}\left[e^{\theta \sum_{k=1}^{n} C_{k}}\right]=e^{c n \theta} \mathrm{E}\left[e^{-c \theta N(n)}\right],
$$

${ }^{4}$ There is no simple explicit form of the dependence between $T_{n}$ and $\vec{l}(n-1)$. For later use in the paper, it sufficient to note that when $N=2$ we have $T_{n}=l_{n-1}$ and if $N>2$ we will proceed through the use of bounds on $T_{n}$ by simple functions of $l_{n-1}$.

${ }^{5}$ Note that it can be proved that $\left(C_{n}\right)_{n \geq 1}$ is a Markov modulated process with respect to a certain Markov chain (see Section IV for more details). We have then [21, pp. 244-246] that $\Psi_{C}(\theta)=\log \left(\lambda_{\max }(\boldsymbol{\Xi}(\theta))\right.$, where $\lambda_{\max }$ is a the largest eigenvalue of a certain matrix $\Xi(\theta)$. Computing analytically this Gärtner-Ellis limit through this relation is not mathematically tractable since a closed form expression for $\lambda_{\max }$ is hard to find. In this paper we will avoid this problem by linking $\Psi_{C}(\theta)$ with $\Psi_{L}(\theta)$ and evaluating the latter quantity instead. 
and therefore

$$
\Psi_{C}(\theta)=c \theta+\Psi_{N}(-c \theta) .
$$

If however $p_{11}<p_{01}$, we obtain

$$
\mathrm{E}\left[e^{\theta \sum_{k=1}^{n} C_{k}}\right]=\mathrm{E}\left[e^{c \theta N(n)}\right],
$$

and therefore

$$
\Psi_{C}(\theta)=\Psi_{N}(c \theta) .
$$

In order to complete the proof we make use of a technical result which relates the large deviations behavior of counting processes and their inverses. This result is due to Glynn and Whitt [23] and states that under some mild conditions (which are satisfied here) the Gärtner-Ellis limit $\Psi_{N}(\theta)$ and the Gärtner-Ellis limit $\Psi_{L}(\theta)$ are related as follows

$$
\Psi_{N}(\theta)=-\Psi_{L}^{-1}(-\theta),
$$

Consequently, combining (12), (14) and (15) we obtain the desired result.

Using this theorem as well as Lemma 1 and 2 we obtain the following corollary.

Corollary 1 (Effective bandwidth). Under stability conditions ${ }^{6}$, the queue length process $Q_{n}$ converges in distribution to a r.v. $Q_{\infty}$ that satisfies

$$
P\left[Q_{\infty}>x\right] \approx \exp \left(-\theta^{*}(a) x\right), \text { for } x \text { relatively large, }
$$

where $\theta^{*}(a)$ is the unique positive solution of the equation

$$
\left\{\begin{array}{ll}
\Psi_{L}((a-c) \theta)+c \theta=0 & \text { if } p_{11} \geq p_{01}, \\
\Psi_{L}(a \theta)-c \theta=0 & \text { o.w. }
\end{array} .\right.
$$

The effective bandwidth for cognitive communications with buffer size $b$ and a maximum buffer overflow probability of $\epsilon$ is given by

$$
a^{*}(\epsilon)=\left\{\begin{array}{ll}
c+\frac{\Psi_{L}^{-1}\left(-c \theta^{*}\right)}{\theta^{*}} & \text { if } p_{11} \geq p_{01}, \\
\frac{\Psi_{L}^{-1}\left(c \theta^{*}\right)}{\theta^{*}} & \text { o.w. }
\end{array} .\right.
$$

where $\theta^{*}=-\frac{\log (\epsilon)}{b}$.

From Corollary 1 we can see that what remains is to obtain a formula for $\Psi_{L}(\cdot)$. We will treat separately the case $N=2$ and the case $N>2$.

\section{The case of $N=2$ channels}

In this section we consider the case where the user can only access 2 channels. In this case the sequence $\left(L_{k}\right)_{k \geq 1}$ is a first-order Markov chain. The following theorem gives a closed form expression for $\Psi_{L}(\cdot)$.

Theorem 2 (Closed-form expression of $\Psi_{L}(\theta)$ ). The sequence $\left(L_{k}\right)_{k \geq 1}$ has the following Gärtner-Ellis limit.

$\Psi_{L}(\theta)=\lim _{n \rightarrow \infty} \frac{\log \mathrm{E}\left[e^{\theta S_{n}}\right]}{n}=\left\{\begin{array}{l}\theta+\log (\Psi(\theta)) \quad \text { if } \theta<\kappa \\ \infty, \text { o.w. }\end{array}\right.$.

${ }^{6}$ See Appendix I. where $\kappa=\log \left(\frac{1}{p_{11}}\right)$ if $p_{11} \geq p_{01}$ and $\kappa=\log \left(\frac{1}{p_{00}}\right)$ otherwise, and

$$
\Psi(\theta)=\frac{\phi_{1}(\theta)+\sqrt{\phi_{1}^{2}(\theta)-4 \phi_{2}(\theta)}}{2},
$$

with

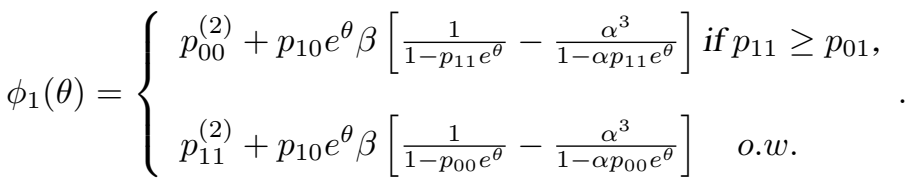

and

$$
\phi_{2}(\theta)=\left\{\begin{array}{ll}
\frac{\beta p_{10} \alpha^{2}(1-\alpha) e^{\theta}}{\left(1-p_{11} e^{\theta}\right)\left(1-\alpha p_{11} e^{\theta}\right)} & \text { if } p_{11} \geq p_{01}, \\
\frac{\beta p_{10} \alpha^{2}(1-\alpha) e^{\theta}}{\left(1-p_{00} e^{\theta}\right)\left(1-\alpha p_{00} e^{\theta}\right)} & \text { o.w. }
\end{array} .\right.
$$

Proof: We give here the proof for the positively correlated case, the proof for the other case is essentially similar and is thus omitted due to space limitations.

In the following, for the sake of notation simplicity, we will abbreviate $\mathrm{E}\left[e^{\theta L_{k}} \mid \vec{L}(k-1)=\vec{l}(k-1)\right]$ by $\mathrm{E}\left[e^{\theta L_{k}} \mid \vec{l}(k-1)\right]$ and $\operatorname{Pr}[\vec{L}(k-1)=\vec{l}(k-1)]$ by $\operatorname{Pr}[\vec{l}(k-1)]$.

In order to see what happens if $\theta \geq \log \left(\frac{1}{p_{11}}\right)$, we fix any arbitrary $n$ and we consider $E\left[e^{\theta S_{n}}\right]$. Due to the Markov property of the sequence $\left(L_{k}\right)_{k \geq 1}$ we have

$$
\mathrm{E}\left[e^{\theta S_{n}}\right]=\sum_{\vec{l}(n-1)} \operatorname{Pr}[\vec{l}(n-1)] e^{\theta \sum_{i=1}^{n-1} l_{i}} \mathrm{E}\left[e^{\theta L_{n}} \mid l_{n-1}\right] .
$$

Notice that $E\left[e^{\theta L_{n}} \mid L_{n-1}=l_{n-1}\right]$ can be calculated as follows $E\left[e^{\theta L_{n}} \mid l_{n-1}\right]=p_{00}^{\left(l_{n-1}+1\right)} e^{\theta}+p_{10} e^{2 \theta} p_{01}^{\left(l_{n-1}+1\right)} \sum_{l_{n}=2}^{\infty}\left(e^{\theta} p_{11}\right)^{l_{n}-2}$,

this expression is hence $\infty$ if $\theta \geq \log \left(\frac{1}{p_{11}}\right)$ and this holds for every fixed $n$, consequently we deduce that $\Psi_{L}(\theta)=\infty$ for $\theta \geq \log \left(\frac{1}{p_{11}}\right)$.

Assume in the following that $\theta<\log \left(\frac{1}{p_{11}}\right)$, then (19) becomes

$$
E\left[e^{\theta L_{n}} \mid l_{n-1}\right]=e^{\theta}\left(1+\frac{p_{01}^{\left(l_{n-1}+1\right)}\left(e^{\theta}-1\right)}{1-p_{11} e^{\theta}}\right) .
$$

Using the fact that $p_{01}^{\left(l_{n-1}+1\right)}=\beta\left(1-\alpha^{l_{n-1}+1}\right)$ and after some algebra we deduce that

$$
\mathrm{E}\left[e^{\theta S_{n}}\right]=g_{1}(\theta) \mathrm{E}\left[e^{\theta S_{n-1}}\right]-g_{2}(\theta) \varphi_{n-1}(\theta),
$$

where $g_{1}(\theta)=e^{\theta}+\beta \frac{e^{\theta}\left(e^{\theta}-1\right)}{1-p_{11} e^{\theta}}, g_{2}(\theta)=\beta \alpha \frac{e^{\theta}\left(e^{\theta}-1\right)}{1-p_{11} e^{\theta}}$ and

$$
\varphi_{n-1}(\theta)=\sum_{\vec{l}(n-1)} \operatorname{Pr}[\vec{l}(n-1)] e^{\theta \sum_{i=1}^{n-1} l_{i}} \alpha^{l_{n-1}} .
$$

By conditioning on $\{\vec{L}(n-2)=\vec{l}(n-2)\}$, the last expression becomes

$$
\varphi_{n-1}(\theta)=\sum_{\vec{l}(n-2)} \operatorname{Pr}[\vec{l}(n-2)] e^{\theta \sum_{i=1}^{n-2} l_{i}} \mathrm{E}\left[e^{\theta^{\prime} L_{n-1}} \mid l_{n-2}\right],
$$


where $\theta^{\prime}=\theta+\log (\alpha)$. Using (20), we obtain after some algebraic manipulations

$$
\varphi_{n-1}(\theta)=g_{1}\left(\theta^{\prime}\right) \mathrm{E}\left[e^{\theta S_{n-2}}\right]-g_{2}\left(\theta^{\prime}\right) \varphi_{n-2}(\theta) .
$$

Multiplying (24) by $g_{2}(\theta)$ and substituting the expression of $g_{2}(\theta) \varphi_{k}(\theta)$ obtained from (21) we obtain the following recurrence relation satisfied by the sequence $\left(\mathrm{E}\left[e^{\theta S_{n}}\right]\right)_{n \geq 1}$.

$$
\mathrm{E}\left[e^{\theta S_{n}}\right]=\chi_{1}(\theta) \mathrm{E}\left[e^{\theta S_{n-1}}\right]-\chi_{2}(\theta) \mathrm{E}\left[e^{\theta S_{n-2}}\right],
$$

where

$$
\chi_{1}(\theta)=g_{1}(\theta)-g_{2}\left(\theta^{\prime}\right),
$$

and

$$
\chi_{2}(\theta)=g_{1}\left(\theta^{\prime}\right) g_{2}(\theta)-g_{2}\left(\theta^{\prime}\right) g_{1}(\theta) .
$$

After some algebraic manipulations it can be shown that $\chi_{1}(\theta)=e^{\theta} \phi_{1}(\theta)$ and $\chi_{2}(\theta)=e^{2 \theta} \phi_{2}(\theta)$, with $\phi_{1}(\theta)$ and $\phi_{2}(\theta)$ are as defined in Theorem 2 .

The characteristic equation associated with the recurrence relation (25) is

$$
x^{2}-\chi_{1}(\theta) x+\chi_{2}(\theta)=0
$$

This equation has 2 roots $x_{ \pm}(\theta)=\frac{\chi_{1}(\theta) \pm \sqrt{\chi_{1}^{2}(\theta)-4 \chi_{2}(\theta)}}{2}$. Leading to the following closed form for $\mathrm{E}\left[e^{\theta S_{n}}\right]^{2}$

$$
\mathrm{E}\left[e^{\theta S_{n}}\right]=A x_{+}^{n}(\theta)+B x_{-}^{n}(\theta) .
$$

Consequently, it is easy to see that $\Psi_{L}(\theta)$ will be given by

$$
\Psi_{L}(\theta)=\lim _{n \rightarrow \infty} \frac{\log E\left[e^{\theta S_{n}}\right]}{n}=\log \left(x_{+}(\theta)\right)=\theta+\log (\Psi(\theta)) .
$$

Remark Note that the constants $A$ and $B$ can be solved by computing $\mathrm{E}\left[e^{\theta S_{1}}\right]$ and $\mathrm{E}\left[e^{\theta S_{2}}\right]$. Although we do not need to solve explicitly for these constants here, it is worth noticing that the quantity $\mathrm{E}\left[e^{\theta S_{1}}\right]$ is given by

$$
\mathrm{E}\left[e^{\theta S_{1}}\right]=e^{\theta}\left(1+\max _{i=1 \ldots N} w_{i}(1) \frac{\left(e^{\theta}-1\right)}{1-p_{11} e^{\theta}}\right),
$$

where $\max _{i=1 \ldots N} w_{i}(1)$ represents the highest belief in the initial slot. Consequently, the final equation (29) obtained for $\mathrm{E}\left[e^{\theta S_{n}}\right]$ depends explicitly on the initial belief. However this dependence fades away when we consider $\Psi_{L}(\theta)$.

\section{The case of $N>2$ channels}

The analysis carried out for the case $N=2$ becomes mathematically intractable when the number of channels increases, thereby making it hard to obtain a closed-form expression for $\Psi_{L}(\theta)$ when $N>2$. Therefore we aim in this section to obtain simple and accurate bounds. These bounds will prove to be very tight for a wide range of the parameters.

Before we proceed we should note here that, as in the case $N=2, \Psi_{L}(\theta)=\infty$ if $\theta \geq \kappa$ (where $\kappa$ is given in Theorem. $2)$. Hence in the following we assume that $\theta<\kappa$. The bounds on $\Psi_{L}(\theta)$ are provided in the following theorem.
Theorem 3 (Bounds on $\Psi_{L}(\theta)$ ). For positively correlated primary traffic $\left(p_{11} \geq p_{01}\right)$ and for $\theta \geq 0$, the function $\Psi_{L}(\theta)$ is bounded as follows

$$
\log \left(e^{\theta}+p_{01}^{(N)} f_{1}(\theta)\right) \leq \Psi_{L}(\theta) \leq \log \left(e^{\theta}+\beta f_{1}(\theta)\right),
$$

where $f_{1}(\theta)=\frac{e^{\theta}\left(e^{\theta}-1\right)}{1-p_{11} e^{\theta}}$. The inequalities are reversed if $\theta<0$.

For the negatively correlated case $\left(p_{11}<p_{01}\right)$ and for $\theta \geq 0$,

$\log \left(f_{2}(\theta)+p_{11}^{(2)} f_{3}(\theta)\right) \leq \Psi_{L}(\theta) \leq \log \left(f_{2}(\theta)+p_{11}^{(2 N-1)} f_{3}(\theta)\right)$,

where $f_{2}(\theta)=\frac{e^{2 \theta} p_{01}}{1-p_{00} e^{\theta}}$ and $f_{3}(\theta)=\frac{e^{\theta}\left(1-e^{\theta}\right)}{1-p_{00} e^{\theta}}$. The inequalities are reversed if $\theta<0$.

Before giving the proof, it should be noticed that the bounds for the positively correlated case are asymptotically tight in $N$. Indeed $p_{01}^{(N)}=\beta\left(1-\alpha^{N}\right)$ and $|\alpha|=\left|p_{11}-p_{01}\right|<1$, hence as $N$ increases the lower bound approaches the upper bound geometrically fast. Also notice that for a fixed $N$, these bounds are tighter if $p_{11}$ is close to $p_{01}$.

Proof: First by conditioning on $\{\vec{L}(n-1)=\vec{l}(n-1)\}$ we obtain

$\mathrm{E}\left[e^{\theta S_{n}}\right]=\sum_{\vec{l}(n-1)} \operatorname{Pr}[\vec{l}(n-1)] e^{\theta \sum_{i=1}^{n-1} l_{i}} \mathrm{E}\left[e^{\theta L_{n}} \mid \vec{l}(n-1)\right]$.

We treat now each case separately, we start by the positively correlated case.

Case 1: $p_{11} \geq p_{01}$.

We can develop $\mathrm{E}\left[e^{\theta L_{n}} \mid \vec{l}(n-1)\right]$ as follows

$$
\begin{aligned}
\mathrm{E}\left[e^{\theta L_{n}} \mid \vec{l}(n-1)\right] & =\sum_{l_{n}=1}^{\infty} \operatorname{Pr}\left[L_{n}=l_{n} \mid \vec{l}(n-1)\right] e^{\theta l_{n}} \\
& =e^{\theta}\left(1+\frac{p_{01}^{\left(J_{n}\right)}\left(e^{\theta}-1\right)}{1-p_{11} e^{\theta}}\right) .
\end{aligned}
$$

Therefore we have

$$
\mathrm{E}\left[e^{\theta S_{n}}\right]=\sum_{\vec{l}(n-1)} e^{\theta \sum_{i=1}^{n-1} l_{i}}\left(e^{\theta}+p_{01}^{\left(J_{n}\right)} f_{1}(\theta)\right) \operatorname{Pr}[\vec{l}(n-1)]
$$

Noticing that $p_{01}^{(N)} \leq p_{01}^{\left(J_{n}\right)}$ and assuming $\theta \geq 0$ we obtain

$$
\left(e^{\theta}+p_{01}^{(N)} f_{1}(\theta)\right) \mathrm{E}\left[e^{\theta S_{n-1}}\right] \leq \mathrm{E}\left[e^{\theta S_{n}}\right] .
$$

Consequently, by induction we obtain

$$
\left(e^{\theta}+p_{01}^{(N)} f_{1}(\theta)\right)^{n-1} \mathrm{E}\left[e^{\theta L_{1}}\right] \leq \mathrm{E}\left[e^{\theta S_{n}}\right] .
$$

From this inequality we deduce that

$$
\log \left(e^{\theta}+p_{01}^{(N)} f_{1}(\theta)\right) \leq \Psi_{L}(\theta) .
$$

Similarly using the fact that $p_{01}^{\left(J_{n}\right)} \leq \beta$ we obtain the upper bound given in the theorem. Note finally that these bounds are reversed if $\theta<0$.

Case 2: $p_{11}<p_{01}$.

As it was remarked in [4], the structure of the myopic policy implies that if $l_{n-1}$ is odd then in the $n$th transmission period 
the CU will switch to the channel sensed at the $(n-2)$ th transmission period, consequently the initial belief at the initiation of the $n$th transmission period is given by $p_{11}^{\left(l_{n-1}+1\right)}$. After some algebra we deduce then the following

$$
\mathrm{E}\left[e^{\theta L_{n}} \mid L_{n-1}=2 k-1, \vec{l}(n-2)\right]=f_{2}(\theta)+p_{11}^{(2 k)} f_{3}(\theta) .
$$

When $l_{n-1}$ is even we have that the belief at the initiation of the $n$th transmission period is upper bounded [4] by $p_{11}^{\left(l_{n-1}+4\right)}$. Thus, if $\theta \geq 0$, this leads to the following

$$
\mathrm{E}\left[e^{\theta L_{n}} \mid L_{n-1}=2 k, \vec{l}(n-2)\right] \geq f_{2}(\theta)+p_{11}^{(2 k+4)} f_{3}(\theta) .
$$

Notice now that $p_{11}^{(2 k+4)} \leq p_{11}^{(2 k)} \leq p_{11}^{(2)}$, therefore

$$
\mathrm{E}\left[e^{\theta L_{n}} \mid \vec{l}(n-1)\right] \geq f_{2}(\theta)+p_{11}^{(2)} f_{3}(\theta) .
$$

This proves the following

$$
\mathrm{E}\left[e^{\theta S_{n}}\right] \geq\left(f_{2}(\theta)+p_{11}^{(2)} f_{3}(\theta)\right) \mathrm{E}\left[e^{\theta S_{n-1}}\right] .
$$

The similar argument used for the positively correlated case leads to the desired lower bound.

To find an upper bound, we use the fact that if $l_{n-1}$ is even then the initial belief at the initiation of the $n$th transmission period is lower bounded by $p_{11}^{\left(l_{n-1}+2 N-3\right)}$. We have therefore

$$
\mathrm{E}\left[e^{\theta L_{n}} \mid L_{n-1}=2 k, \vec{l}(n-2)\right] \leq f_{2}(\theta)+p_{11}^{(2(N+k)-3)} f_{3}(\theta) .
$$

Since $p_{11}^{(2 k)} \geq p_{11}^{(2(N+k)-3)} \geq p_{11}^{(2 N-1)}$, we deduce that

$$
\mathrm{E}\left[e^{\theta L_{n}} \mid \vec{l}(n-1)\right] \leq f_{2}(\theta)+p_{11}^{(2 N-1)} f_{3}(\theta) .
$$

Consequently

$$
\mathrm{E}\left[e^{\theta S_{n}}\right] \leq\left(f_{2}(\theta)+p_{11}^{(2 N-1)} f_{3}(\theta)\right) \mathrm{E}\left[e^{\theta S_{n-1}}\right] .
$$

The rest of the proof is similar to the positively correlated case. Note finally that the inequalities will be reversed if $\theta<0$.

Corollary 2. Let $\theta^{*}(a)$ be the stationary decay rate of the queue of the $C U$ when there are $N$ channel. Then we have

$$
\theta_{l}^{*}(a) \leq \theta^{*}(a) \leq \theta_{u}^{*}(a),
$$

where $\theta_{l}^{*}(a)$ and $\theta_{u}^{*}(a)$ are the unique positive solutions of the following equations

Case 1: $p_{11} \geq p_{01}$.

$$
\left(1-p_{11} e^{(a-c) \theta}\right)\left(e^{a \theta}-1\right)+e^{(a-c) \theta}\left(e^{a \theta}-e^{c \theta}\right) x=0,
$$

where the parameter $x$ is such as $x=p_{01}^{(N)}$ for $\theta_{l}^{*}(a)$ and $x=\beta$ for $\theta_{u}^{*}(a)$.

Case 2: $p_{11}<p_{01}$.

$$
e^{a \theta}\left(e^{a \theta}-1\right)(1-x)+\left(e^{a \theta}-e^{c \theta}\right)\left(1-p_{00} e^{a \theta}\right)=0,
$$

where the parameter $x$ is such as $x=p_{11}^{(2)}$ for $\theta_{l}^{*}(a)$ and $x=$ $p_{11}^{(2 N-1)}$ for $\theta_{u}^{*}(a)$.

Proof: The proof is based on the bounds established above on $\Psi_{L}(\theta)$.

\section{NUMERICAL SimULATION}

We conduct numerical simulations to validate the derived expressions. Given that we are interested in the regime that the overflow probability is very small, standard Monte Carlo (MC) simulation techniques are inadequate for our purposes. We resort therefore to the use of importance sampling techniques. The problem considered in this paper has a nice structure that makes it possible to use the importance sampling tools derived for Markov modulated processes in [21].

\section{A. Simulation via Importance Sampling Techniques}

At each time slot $n$, define a vector $\mathbf{Z}_{n}$ as $\mathbf{Z}_{n}=\left(\mathbf{X}_{n}, I_{n}\right)$, where $\mathbf{X}_{n}=\left(X_{n}(1), X_{n}(2), \ldots, X_{n}(N)\right) \in\{0,1\}^{N}$ represents the state of the $N$ different channels and $I_{n} \in\{1, \ldots N\}$ denotes the channel being sensed in the $n$th slot. Under the myopic policy (the ZKL protocol), it can be seen that the process $\left(\mathbf{Z}_{n}\right)_{n \geq 1}$ forms a Markov chain on $\mathcal{S}=\{0,1\}^{N} \times$ $\{1, \ldots N\}$ with a certain transition matrix $\mathbf{R}$. It can be verified that the random output process $\left(C_{n}\right)_{n \geq 1}$ of the queue of the $\mathrm{CU}$ is a Markov Modulated Process (MMP) with respect to $\left(\mathbf{Z}_{n}\right)_{n \geq 1}$, indeed $C_{n}=F\left(\mathbf{Z}_{n}\right) \triangleq c \mathbf{X}_{n}\left(I_{n}\right)$. This formulation of the problem allows us to simulate the queue of the $\mathrm{CU}$ and obtain estimates for $P\left(Q_{\infty}>x\right)$ for large $x$ using the techniques described in [21].

To be more specific, assume that the system has been running for a long time (say from $t=-\infty$ ) so that stationarity has been reached at time $t=0$. We have then $P\left(Q_{\infty}>x\right)=\frac{\mathrm{E}[Y]}{\mathrm{E}\left[H_{0}\right]}$ (see e.g., [25]), where $H_{0}$ is the first time the queue empties and $Y=\sum_{n=1}^{H_{0}-1} 1_{\left\{Q_{n}>x\right\}}$. The denominator $\mathrm{E}\left[H_{0}\right]$ can be estimated using standard MC simulations but $\mathrm{E}[Y]$ requires importance sampling with a biased Markov process. To this end, enumerate $\mathcal{S}$ as $\left\{\mathbf{s}_{1}, \mathbf{s}_{2}, \ldots, \mathbf{s}_{|\mathcal{S}|}\right\}$ and let $\boldsymbol{\Gamma}(\theta)=\operatorname{diag}\left(\gamma_{1}(\theta), \ldots, \gamma_{|S|}(\theta)\right)$ where $\gamma_{i}(\theta)=\exp \left(\theta F\left(\mathbf{s}_{i}\right)\right)$. The biased Markov process (still on $\mathcal{S}$ ) used for simulations is $\left(\widetilde{\mathbf{Z}}_{n}\right)_{n \geq 1}$ with a transition matrix $\widetilde{\mathbf{R}}$ where $\widetilde{R}_{i j}=\frac{R_{i j} \gamma_{j}\left(\theta^{*}\right) \eta_{j}\left(\theta^{*}\right)}{\eta_{i}\left(\theta^{*}\right) \lambda_{\max }\left(\mathbf{R} \Gamma\left(\theta^{*}\right)\right)}, \lambda_{\max }\left(\mathbf{R} \boldsymbol{\Gamma}\left(\theta^{*}\right)\right)$ is the maximal eigenvalue of $\mathbf{R} \boldsymbol{\Gamma}\left(\theta^{*}\right),\left(\eta_{1}\left(\theta^{*}\right), \ldots, \eta_{|\mathcal{S}|}\left(\theta^{*}\right)\right)^{T}$ is the corresponding right eigenvector and $\theta^{*}$ is the unique positive solution to the equation $a \theta=-\log \left(\lambda_{\max }(\mathbf{R} \Gamma(-\theta))\right)$. Then we have $\mathrm{E}[Y]=\widetilde{\mathrm{E}}[\mathcal{L} Y]$ where $\widetilde{\mathrm{E}}$ denotes the expectation with respect to $\left(\widetilde{\mathbf{Z}}_{n}\right)_{n \geq 1}$ and $\mathcal{L}$ is the likelihood ratio defined, for a sample path $\omega=\left(\mathbf{s}_{i_{1}}, \ldots, \mathbf{s}_{i_{m}}\right)$, as $\mathcal{L}(\omega)=\frac{\eta_{\mathbf{s}_{i_{1}}}\left(\theta^{*}\right)}{\eta_{\mathbf{s}_{i_{m}}}\left(\theta^{*}\right)}\left(\lambda_{\max }\left(\mathbf{R} \boldsymbol{\Gamma}\left(\theta^{*}\right)\right)\right)^{m} \exp \left(-\theta^{*} \sum_{k=1}^{m} F\left(\mathbf{s}_{i_{k}}\right)\right)$. The expectation $\widetilde{\mathrm{E}}[\mathcal{L} Y]$ can be finally computed using standard MC simulations since when the system is simulated with $\left(\widetilde{\mathbf{Z}}_{n}\right)_{n \geq 1}$, the event $\left\{Q_{\infty}>x\right\}$ is not rare anymore. For further details on importance sampling for MMPs the interested reader is referred to [21, Section 9.6 and 9.7].

\section{B. Simulation Setup and Results}

For the plots provided in this paper, the channel parameters used are summarized in Table I. The set A describes a positively correlated scenario while the set $\mathrm{B}$ describes a negatively correlated one. Throughout this simulation section $c$ is taken to be 1 . 


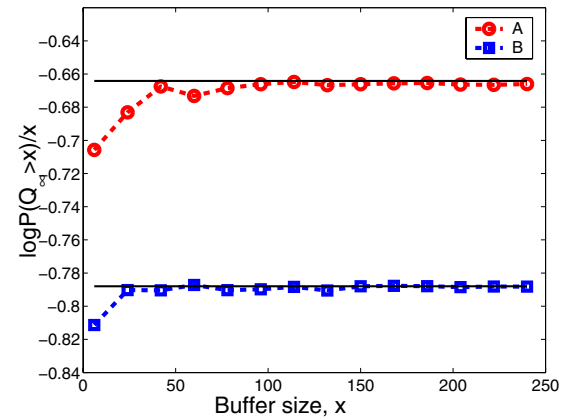

(a) $\log P\left(Q_{\infty}>x\right) / x$ vs Buffer size

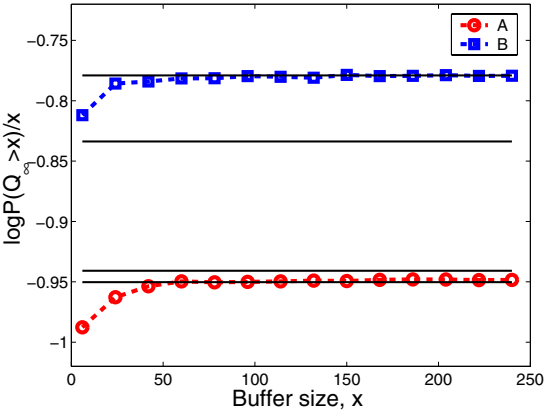

(a) $\log P\left(Q_{\infty}>x\right) / x$ vs Buffer size

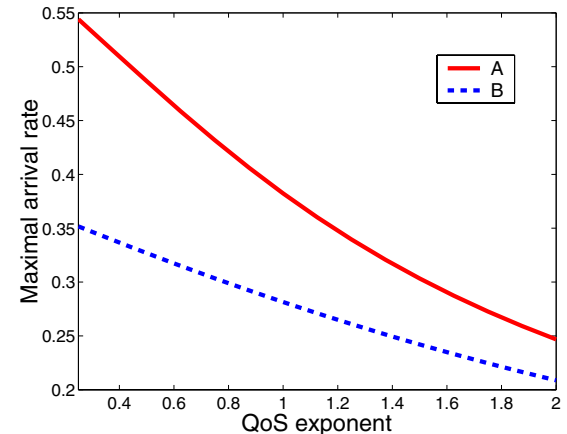

(b) Effective capacity vs QoS exponent

Fig. 2. Two channels

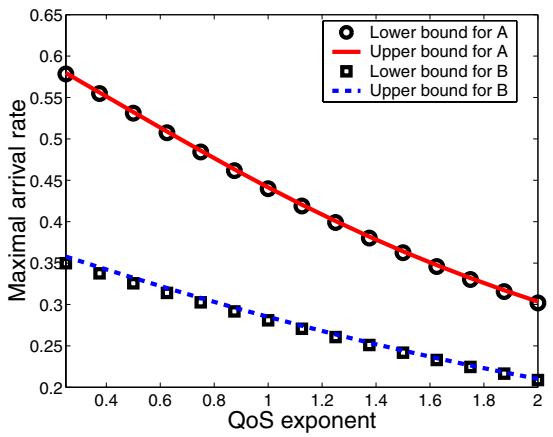

(b) Effective capacity vs QoS exponent

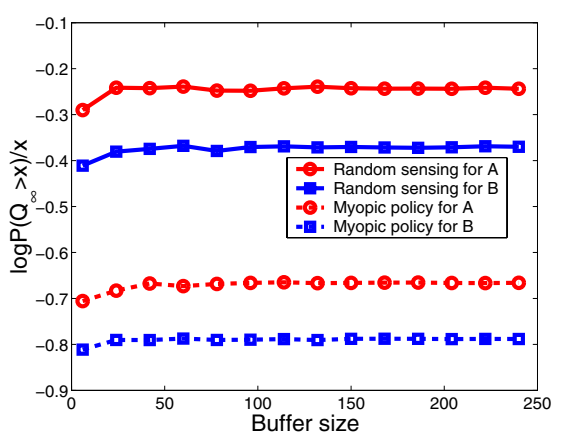

(c) Random sensing vs myopic policy

Fig. 3. Six channels

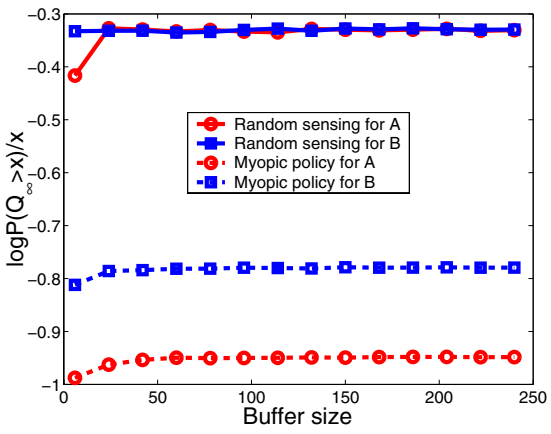

(c) Random sensing vs myopic policy

\begin{tabular}{|c|c|c|c|}
\hline parameter set & $p_{11}$ & $p_{01}$ & $a$ \\
\hline $\mathrm{A}$ & 0.7 & 0.3 & 0.45 \\
\hline $\mathrm{B}$ & 0.2 & 0.4 & 0.3 \\
\hline
\end{tabular}

TABLE I. Channel parameters

Fig. 2(a) depicts the quantity $\log P\left(Q_{\infty}>x\right) / x$ obtained via simulation (in dashed lines) versus the buffer size $x$ for the two channels case. For comparison, the quantity $-\theta^{*}(a)$ obtained from solving the equations provided in Corollary 1 (with the expressions of $\Psi_{L}(\theta)$ given in Theorem 2) is also given in solid lines. The plots demonstrate that $\log P\left(Q_{\infty}>\right.$ $x) / x$ converges to the theoretically computed limit $-\theta^{*}(a)$ as $x$ increases. From the plots it can be seen that the convergence happens relatively fast and this is true even for moderate buffer sizes (i.e., $x \geq 30$ ). This plot validates the theoretically computed expressions of $\Psi_{L}(\theta)$ given in Theorem 2 .

Fig. 3(a) shows the same quantities but for 6 channels. The upper and lower bounds of $-\theta^{*}(a)$ plotted with solid lines are those obtained from solving the equations in Corollary 2. In the positive correlation case the bounds prove to be particularly tight. In the negative correlation case the upper bound for $-\theta^{*}(a)\left(-\theta_{l}^{*}(a)\right)$ is close to the simulated curve however the lower bound $\left(-\theta_{u}^{*}(a)\right)$ proves to be loose. We should note at this point that we were able to obtain more accurate bounds. However, the expressions and the derivations of these bounds are more complicated and the space limitations preclude the communication of these results in this paper.

We now turn to the problem of evaluating the maximal arrival rate. In the following, we provide plots showing the maximal arrival rate $a^{*}(\epsilon)$ versus the QoS exponent $-\frac{\log (\epsilon)}{b}$. We fix a buffer of size $b=40$, which from the previous plots suggests that the approximation $P\left(Q_{\infty}>b\right) \approx e^{-\theta^{*}(a) b}$ is relatively accurate. We then depict $a^{*}(\epsilon)$ by varying $\epsilon$. When $N=2$, through the relation given in Corollary 1 , the closed form expression for $\Psi_{L}(\theta)$ (c.f. Theorem 2) is used to plot $a^{*}(\epsilon)$ in Fig. 2(b). When $N=6$, the bounds for $\Psi_{L}(\theta)$ obtained in Theorem 3 are used to generate the plots provided in Fig. 3(b). As it can be seen the upper and lower bounds provided are close to each other suggesting that they are close to the true value. By looking at Figs. 2(b) and 3 (b), one can notice that in the negatively correlated case, the maximal arrival rate seems to be unaffected by an increase in the number of channels. The same is not true for the positively correlated case since increasing the channels from 2 to 6 shows an improvement in the maximal arrival rate.

In Figure 2(c) and 3(c) we compare the decay rates of the $\mathrm{CU}$ with two different access policies; the myopic policy and a random access policy. At the beginning of each slot, the random access policy senses uniformly randomly one of the $N$ channels. As such the random access policy is completely memoryless and totally ignores the history of the sensing outcomes in the previous slots. One would expect therefore 
that the random access policy performs poorly when compared to the myopic policy. This is confirmed by the plots since as it can be seen the decay rates for the random access policy are inferior to the decay rates obtained with the myopic policy.

\section{CONCLUSIONS}

In this paper we have investigated the queueing behavior of cognitive communications. Within a multi-channel opportunistic communication framework, a cognitive user $(\mathrm{CU})$ is using a myopic sensing scheme to sense one out of $N$ independent primary ON-OFF channels. For this problem, we have studied the decay rate of the tail distribution of the CU's queue. When $N=2$, we have provided a complete characterization of the decay rate in terms of the system parameters. When $N>2$, we have obtained upper and lower bounds on the decay rate. The expressions that we have derived have been used to determine the maximum arrival rate that the $\mathrm{CU}$ can support while the buffer overflow probability is kept under a predefined QoS guarantee.

There are several future directions that we wish to pursue such as the determination of the decay rate when the $\mathrm{CU}$ is capable of sensing multiple channels in each slot. A possible extension include also the study of the decay rate with different sensing policies.

\section{APPENDIX I}

We give here some considerations to the stability of the queue of the $\mathrm{CU}$. We do this by considering the embedded sequence $\left(Q_{n}^{e}\right)_{n \geq 1}$ defined as $Q_{n}^{e}=Q_{S_{n}}$. In other words the sequence $\left(Q_{n}^{e}\right)_{n \geq 1}$ is the queue length at the end of the $n$th transmission period. This sequence satisfies the recursion $Q_{n}^{e}=\left(Q_{n-1}^{e}+U_{n}\right)^{+}$where $U_{n}=(a-c) L_{n}+c$ if $p_{11} \geq p_{01}$ and $U_{n}=a L_{n}-c$ otherwise. The queue is said to be stable if [24]

$$
\bar{U} \triangleq \lim _{n \rightarrow \infty} \frac{\sum_{i=1}^{n} U_{i}}{n}<0 \quad \text { a.s. }
$$

Consequently, it can be seen that in the positively correlated case, the queue is stable if $\bar{L}>\frac{c}{c-a}$ whereas if $p_{11}<p_{01}$ the queue is stable if $\bar{L}<\frac{c}{a}$. The average length of a transmission period $\bar{L}$ was studied in [4]. For $N=2$ closedform expressions were obtained, whereas for $N>2$ upper and lower bounds for $\bar{L}$ were provided. Using these expressions we get several conditions on the stability of the queue of the CU. For instance, in the positively correlated case, when $N=2$ the queue is stable if

$$
\beta\left(1-\alpha^{2}\right)(a-c)+a p_{10}\left(1-e_{2}\right)<0,
$$

where $e_{2}=\frac{p_{01} \alpha^{2}}{1-\alpha p_{11}}$.Also as $N \rightarrow \infty$, when $p_{11} \geq p_{01}$, the queue is stable if

$$
\frac{\beta(a-c)}{1-p_{11}}+a<0
$$

\section{REFERENCES}

[1] Q. Zhao and B. M. Sadler, "A survey of dynamic spectrum access," IEEE Signal Processing Magazine, vol. 55, no. 5, pp. 2294-2309, May, 2007.

[2] Q. Zhao, L. Tong, A. Swami, and Y. Chen, "Decentralized cognitive MAC for opportunistic spectrum access in Ad Hoc networks: a POMDP framework," IEEE JSAC, vol. 25, no. 3, pp. 589-600, April, 2007.

[3] F. P. Kelly, Notes on Effective Bandwidths, in Stochastic Networks, Clarendon Press, Oxford, pp. 141-168, 1996.

[4] Q. Zhao, B. Krishnamachari, and K. Liu "On Myopic Sensing for MultiChannel Opportunistic Access: Structure, Optimality, and Performance" IEEE Transactions on Wireless Communications, vol. 7, no. 12, pp. 54315440, December, 2008.

[5] S.H. Ahmad, M. Liu, T. Javidi, Q. Zhao, and B. Krishnamachari, "Optimality of Myopic Sensing in Multi-Channel Opportunistic Access," to appear in IEEE Transactions on Information Theory.

[6] P. W. Glynn and W. Whitt, "Logarithmic Asymptotics for SteadyState Tail Probabilities in a Single-Server Queue," Journal of Applied Probability, pp. 131-155, 1994.

[7] J. Abate, G. L. Choudhury, W. Whitt, "Exponential Approximations for Tail Probabilities in Queues, I and II" Operations Research, 1995 and 1996.

[8] G. de Veciana, J. C. Walrand, "Effective bandwidths: Call admission, traffic policing and filtering for ATM networks," Queueing Systems vol. 20, pp. 37-59, 1995.

[9] A. Elwalid, D. Mitra, "Effective Bandwidth of General Markovian Traffic Sources and Admission Control of High Speed Networks," IEEE/ACM Transactions on Networking, vol. 1, no. 3, pp. 329-343, 1993.

[10] G. Kesidis, J. Walrand, and C. Chang, "Effective Bandwidths for Multiclass Markov Fluids and Other ATM Sources," IEEE/ACM Transactions on Networking, vol. 1, no. 4, pp. 424-428, Aug. 1993.

[11] C. -S. Chang, "Stability, Queue Length, and Delay of Deterministic and Stochastic Queueing Networks," IEEE Trans. Auto. Control, vol. 39, no. 5, pp. 913-931, May 1994.

[12] D. Tse, R. G. Gallager and J. N. Tsitsiklis, "Statistical Multiplexing of Multiple Time-scale Markov Streams," IEEE Journal on Selected Areas in Communications, vol. 13, no. 6., pp. 1028-1039, Aug. 1995.

[13] D. Wu and R. Negi, "Effective capacity: A wireless link model for support of quality of service," IEEE Trans. Wireless Commun., vol. 2, no. 4, pp. 630-643, July 2003.

[14] L. Liu, P. Parag, J. Tang, W.-Y. Chen and J.-F. Chamberland, "Resource Allocation and Quality of Service Evaluation for Wireless Communication Systems Using Fluid Models," IEEE Transactions on Information Theory, vol. 53, no. 5, pp. 1767-1777, May 2007.

[15] L. Ying, R. Srikant, A. Eryilmaz, and G. E. Dullerud, "A Large Deviations Analysis of Scheduling in Wireless Networks," IEEE Transactions on Information Theory, vol. 52, no. 11, November 2006.

[16] S. Shakkottai, "Effective Capacity and QoS for Wireless Scheduling," IEEE Transactions on Automatic Control, vol. 53, no. 3, April 2008.

[17] S. Wang, J. Zhang and L. Tong, "Delay Analysis for Cognitive Radio Networks with Random Access: A Fluid Queue View," submitted to INFOCOM'10.

[18] S. Akin, and M. C. Gursoy, "Effective Capacity Analysis of Cognitive Radio Channels for Quality of Service Provisioning," Submitted June 2009 (http://arxiv.org/abs/0906.3888).

[19] Z. Fan, "Performance Analysis of Dynamic Spectrum Access Networks," in Proc. IEEE International Conference on Pervasive Computing and Communications, 2008, pp. 336-341.

[20] M. M. Rashid, J. Hossain, E. Hossain, and V. K. Bhargava, "Opportunistic Spectrum Access in Cognitive Radio Networks: A Queueing Analytic Model and Admission Controller Design" in Proc. IEEE GLOBECOM 2007, pp. 4647-4652.

[21] C. -S. Chang, "Performance Guarantees in Communications Networks," Springer Verlag, 2000.

[22] L. Liu, and J.-F. Chamberland, "On The Effective Capacities of MultipleAntenna Gaussian Channels," in IEEE International Symposium on Information Theory, pp. 2583-2587, 2008

[23] P. W. Glynn and W. Whitt, "Large Deviations Behavior of Counting Processes and Their Inverses," Queueing Systems, vol. 17, 1994, pp. $107-$ 128.

[24] A. A. Borokov, "Stochastic Processes in Queueing Theory," Springer Verlag, New York, 1976.

[25] R. Cogburn "A unified theory for sums of Markov chain transition probabilities," The Annals of Probability, vol. 3, pp. 191-214, 1975. 